\title{
Long range millimeter wave wireless links enabled by traveling wave tubes and resonant tunnelling diodes
}

\author{
Claudio Paoloni ${ }^{1}$, Rupa Basu ${ }^{1}$, Laxma R. Billa ${ }^{1}$, Jeevan N. Rao ${ }^{1}$, Rosa Letizia ${ }^{1}$, Qiang Ni ${ }^{2}$, Edward Wasige ${ }^{3}$, \\ Abdullah Al-Khalidi ${ }^{3}$ and Jue Wang ${ }^{3}$ \\ ${ }^{1}$ Engineering Department, Lancaster University, Lancaster, UK \\ email: c.paoloni@lancaster.ac.uk \\ ${ }^{2}$ School of Computing and Communications, Lancaster University, Lancaster, UK \\ ${ }^{3}$ High Frequency Electronics Group, School of Engineering, University of Glasgow, Glasgow, UK
}

\begin{abstract}
This paper describes a new project to realize a high data rate point to point wireless system above $150 \mathrm{GHz}$. The upper end of the D-band spectrum is used (151 -174 GHz) for full duplex transmission. The aims it to enable a full fiber on air with more than $1 \mathrm{~km}$ range to provide up to $45 \mathrm{~Gb} / \mathrm{s}$ data rate. The system consists in a transmitter using a directly modulated Resonant Tunnelling Diode (RTD) oscillator and powered by novel traveling wave tubes (TWT).
\end{abstract}

Keywords-millimeter waves, D-band, links, wireless, TWT, RTD, Point-to-point, capacity

\section{INTRODUCTION}

The congestion of existing mobile networks, the crucial role of data in the fourth industrial revolution and new datahungry applications such as augmented reality, real-time remote medicine, car-to-car communications imply a revolution in the distribution of internet beyond the fibre. It is predicted that videos will account for $75 \%$ of the full mobile traffic by 2023 and, by 2021, PCs will account for only $25 \%$ of traffic [1]. This new scenario requires high complexity and densification of networks both for mobile and fixed access, with very high data rate backhaul [2]. Multi-gigabit per second $(\mathrm{Gb} / \mathrm{s})$ data rate wireless distribution is emerging as the most affordable solution to satisfy data demand and complement fibre, that often has high deployment costs. The millimetre waves (mm-waves) portion of the spectrum offers the wide frequency bands needed for multi-Gb/s transmission. Presently, most of the available bands below $100 \mathrm{GHz}$ are already used. Point to point (PtP) links up to E-band (71-76 $\mathrm{GHz}, 81-86 \mathrm{GHz}$ ) are in the market. Following this move up in frequency, the need for higher and higher capacity can be solved. Millimeter-waves above $100 \mathrm{GHz}$, where more than $100 \mathrm{GHz}$ of fragmented frequency bands are still not exploited, can support tens of $\mathrm{Gb} / \mathrm{s}$, as demonstrated by the research in solid state transmitters up to $400 \mathrm{GHz}[3,4]$. In particular, the D-band $(141-174.5 \mathrm{GHz})$, with a total of 28 $\mathrm{GHz}$ divided in three sub bands, is attracting the interest of big manufacturers and the attention of the main standardization and regulatory bodies, OFCOM, FCC (US), CEPT (Europe), ETSI [5] for paving the way for its commercial exploitation

\section{Challenges}

Above $100 \mathrm{GHz}$, the high atmosphere and rain attenuation and the low transmission power of solid-state amplifiers, make it arduous to build wireless links with adequate range and with the $99.99 \%$ availability required by telecom operators, unless very simple modulation schemes are used due to the sharp reduction in signal to noise ratio (SNR) with distance. That prevents the use of high-order modulation schemes (e.g. 64 QAM - quadrature amplitude modulation) or higher. For instance, below $100 \mathrm{GHz}$, E-band links are adverted to reach theoretically $10 \mathrm{~Gb} / \mathrm{s}$ data rate with $2 \mathrm{GHz}$ bandwidth, but probably in clear sky. If $99.99 \%$ availability, required to operate in common ITU rain zones, is considered, a substantial reduction of performance is expected. In the case of D-band, the total link losses (ITU zone K, $42 \mathrm{~mm} / \mathrm{h}$ rain) at upper frequency $(175 \mathrm{GHz})$, for $1 \mathrm{~km}$ distance, are about 160 dB, against e.g. $130 \mathrm{~dB}$ at $28 \mathrm{GHz}$. A PtP link needs about 40 $\mathrm{dBm}(10 \mathrm{~W})$ saturated transmission power for 64 QAM. No solid-state power amplifier (PA) can provide this high power. $\mathrm{GaN}$ PAs at $100 \mathrm{GHz}$ reach $30-32 \mathrm{dBm}$. D-band InP technology PAs could reach $16-19 \mathrm{dBm}$ [6]. Therefore, the exploitation of mm-wave spectrum above $100 \mathrm{GHz}$ needs a breakthrough in transmission power.

\section{D-BAND POINT TO POINT}

The DLINK project aims to demonstrate the first D-band wireless link, unprecedented data rate up to $45 \mathrm{~Gb} / \mathrm{s}$, over 1 $\mathrm{km}$ distance, with $99.99 \%$ availability, in ITU zone K, for high capacity wireless networks (Fig.1). It will be built by integrating for the first time RTDs (Resonant Tunnelling Diodes) and TWTs (Traveling Wave Tubes) to exploit their complementarity in a novel mm-wave system.

\section{A. System}

DLINK system consists of two front ends, one for transmission in the Upper Band with receiver in Lower Band, the second, for transmission in Lower Band with receiver in Upper Band (Fig.2). Each front end includes one vector

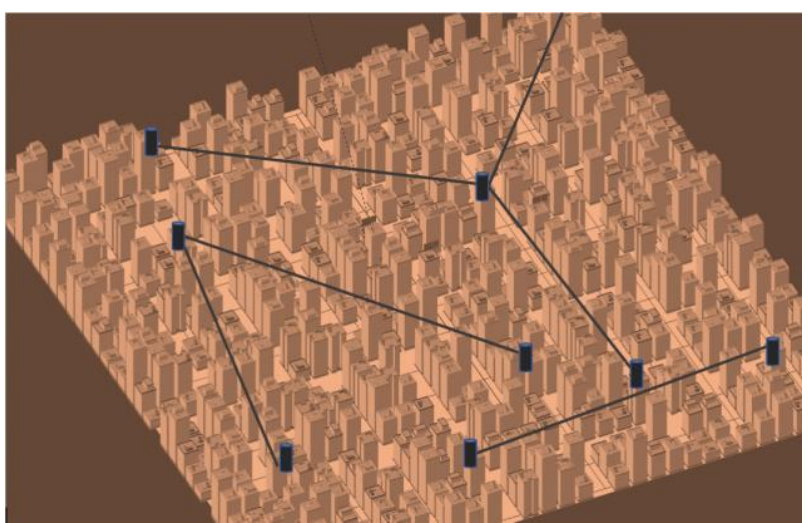

Fig. 1. DLINK concept 


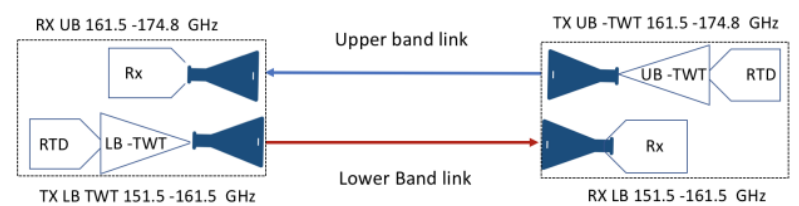

Fig. 2. DLINK system schematic

modulator, one RTD, one TWT, and one receiver. Antennas with about $38 \mathrm{dBi}$ gain will be used. Two novel TWTs, one in $151.5-161.5 \mathrm{GHz}$ band and one in $161.5-174.8 \mathrm{GHz}$ band, for each front end, will be designed and built. The two front ends covering the $20 \mathrm{GHz}$ useful bandwidth will provide for the first time a wide band link in FDD, for long distance, for new insight in D-band propagation.

\section{B. Specifications}

The target is to provide fiber data rate level wireless for more than $1 \mathrm{~km}$, exploiting the wide frequency band above $150 \mathrm{GHz}$.

Ten 1-GHz channels will be considered. If 64 QAM 5/6 is assumed, each channel can provide about $4.5 \mathrm{~Gb} / \mathrm{s}$ data rate, for a total $45 \mathrm{~Gb} / \mathrm{s}$.

\section{D-band Travelling Wave Tubes}

Travelling Wave Tubes (TWTs) are wide band vacuum electronic amplifiers widely used in microwave communications. A TWT (Fig. 3) is made by an electron gun, a slow wave structure (SWS), a collector, a magnetic focusing system and vacuum RF windows to seal the flanges. The electron gun generates an electron beam with given current and voltage, confined by the magnetic system along the SWS. The SWS permits the transfer of energy from the beam to the input RF fields, by slowing down the field phase velocity close to the speed of the electrons. This mechanism permits to achieve multi-Watt power at mm-waves making TWTs the only solution for mm-waves high power amplification. Helix SWSs, typical of microwaves, have excellent performance, but are not feasible above $70 \mathrm{GHz}$ due to the small dimensions required. Due to high cost and technology challenges, TWTs above $100 \mathrm{GHz}$ are not available in the market and only a few prototypes have been built [7]. The objective is to build low cost TWTs based on the double corrugated waveguide, already demonstrated easy to build by conventional CNC machining and with good electrical performance [8,9].

\section{Resonant Tunnelling Diodes}

Resonant tunnelling diodes (RTDs) are the fastest electronic devices with demonstrated frequencies of up to 1.98 $\mathrm{THz}$ [10]. State of the art RTD based transmitters include 2 $\mathrm{mW}, 15 \mathrm{Gbps}$ at W-band using on-off keying (OOK) and amplitude shift keying (ASK) [11] and $1 \mathrm{~mW}$ with $110 \mathrm{GHz}$ modulation bandwidth at $260 \mathrm{GHz}$ [12]. The fastest reported wireless data rates using RTD based transmitters include 34 Gbps at $500 \mathrm{GHz}$ using single channel and $56 \mathrm{Gbps}$ using dual channel links [13]-[14].

On this project, we are developing D-band RTD transmitters with integrated vector modulators as TWT drivers. The use of a higher order modulation scheme such as quadrature amplitude modulation (QAM) is crucial to meeting the target data rates. To do this, very low-phase noise oscillators $(<-90 \mathrm{dBc} / \mathrm{Hz}$ at $100 \mathrm{kHz}$ offset and $<-110 \mathrm{dBc} / \mathrm{Hz}$ at $1 \mathrm{MHz}$ offset) are needed and are being developed. A key advantage of this approach is the lack of a mixer and an amplifier from the transmitter, and this further enhances the low phase noise oscillator properties required for QAM.

At the heart of a vector modulator is a Lange coupler in a reflection topology with PIN diodes or FETs acting as switches on the coupled and direct ports [15]. Such a modulator which employs InP-based InGaAs PIN diodes [16] is being developed on this project at D-band. Since both RTDs and PIN diodes can be realised from the same InP-based material system, therefore through hetero-epitaxial integration (Fig.4) the monolithic integration of the modulator and transmitter will be implemented. Thus, the ultimate goal is the demonstration of an InP-based RTD/PIN diode technology as a low cost wireless transmitter technology, and one which can be used to drive a TWT.

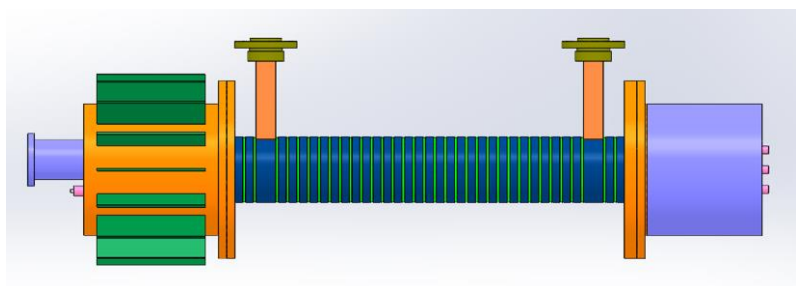

Fig. 3. Traveling wave tube

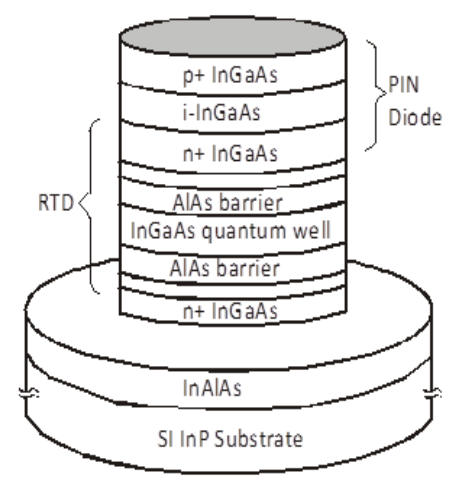

Fig. 4. Resonant tunnel diode (RTD) and PIN diode

\section{CONCLUSIONS}

The availability of the high capacity point to point links will allows to connect sections of networks where the fiber cannot be deployed. The DLINK project aims to produce innovative front end at D-band to exploit the wide band to transmit multigigabit data rate. The components of the DLINK system are in advanced design stage.

\section{ACKNOWLEDGMENT}

The DLINK- D-band Wireless Link with Fibre Data Rate project is funded by EPSRC grants EP/S009620/1 and EP/S009442/1.

\section{REFERENCES}

[1] Cisco Visual Networking Index: Global Mobile Data Traffic Forecast Update, 2016-2021 White Paper, 2017

[2] J. Shi, L. Lv, Q. Ni, H. Pervaiz, C. Paoloni. "Modeling and Analysis of Point-to-Multipoint Millimeter-Wave Backhaul Networks", IEEE 
Transactions on Wireless Communications, Vol. 18, Issue 1, pp. 268 285, January 2019.

[3] Nagatsuma, Tadao, Guillaume Ducournau, and Cyril C. Renaud. "Advances in terahertz communications accelerated by photonics." Nature Photonics 10, no. 6, pp. 371-379, 2016.

[4] Dhillon, S. S., et al. "The 2017 terahertz science and technology roadmap." Journal of Physics D: Applied Physics, 50.4, pp. 043001, 2017).

[5] Lombardi, R. "Microwave and Millimetre-wave for 5G Transport." ETSI White Paper 25, February. 2018

[6] Merkle, T., et al. "Compact 110-170 GHz amplifier in $50 \mathrm{~nm}$ mHEMT technology with $25 \mathrm{~dB}$ gain." European Microwave Integrated Circuit Conference (EuMIC), pp. 129-132, IEEE, 2013.

[7] L. Wenqiang et al., "Development of D-band continuous-wave folded waveguide traveling-wave tube," 2015 IEEE International Vacuum Electronics Conference (IVEC), pp.1-3, IVEC.2015.

[8] M. Mineo and C. Paoloni, "Double Corrugation Rectangular Waveguide Slow-wave Structure for THz Vacuum Devices”, IEEE Trans. on Electron Devices, pp.3169-3175, November 2010.

[9] Paoloni, C et al., TWEETHER Future Generation W-band backhaul and access network infrastructure and technology. in European Conference on Networks and Communications (EuCNC 2017), pp. 1 5, IEEE, 2017.

[10] R. Izumi, S. Suzuki and M. Asada, "1.98 THz resonant-tunneling-diode oscillator with reduced conduction loss by thick antenna electrode." 42nd International Conference on Infrared, Millimeter, and Terahertz Waves (IRMMW-THz), 2017.
[11] J. Wang, A. Al-Khalidi, L. Wang, R. Morariu, A. Ofiare and E. Wasige, "15-Gb/s 50-cm Wireless Link Using a High-Power Compact III-V 84GHz Transmitter," in IEEE Transactions on Microwave Theory and Techniques, vol. 66, no. 11, pp. 4698-4705, Nov. 2018.

[12] A. Al-Khalidi, J. Wang and E. Wasige, "Compact J-band Oscillators With $1 \mathrm{~m}$ RF output Power and Over $110 \mathrm{GHz}$ Modulation Bandwidth," 2018 43rd International Conference on Infrared, Millimeter, and Terahertz Waves (IRMMW-THz), Nagoya, pp. 1-2, 2018.

[13] N. Oshima, K. Hashimoto, S. Suzuki, and M. Asada, "Wireless data transmission of $34 \mathrm{Gbit} / \mathrm{s}$ at a $500-\mathrm{GHz}$ range using resonant tunnelling diode terahertz oscillator," Electron. Lett., vol. 52, no. 22, pp. 18971898, Oct. 2016.

[14] N. Oshima, K. Hashimoto, S. Suzuki and M. Asada, "Terahertz Wireless Data Transmission With Frequency and Polarization Division Multiplexing Using Resonant-Tunneling-Diode Oscillators," in IEEE Transactions on Terahertz Science and Technology, vol. 7, no. 5, pp. 593-598, Sept. 2017.

[15] A. E. Ashtiani, Sueng-Il Nam, A. d'Espona, S. Lucyszyn and I. D. Robertson, "Direct multilevel carrier modulation using millimeterwave balanced vector modulators," in IEEE Transactions on Microwave Theory and Techniques, vol. 46, no. 12, pp. 2611-2619, Dec. 1998.

[16] V. Ziegler et al, "InP-based monolithic integrated PIN diode switches for mm-wave applications," GaAs Mantech, 1998. 